\title{
Théologiques
}

Revue interdisciplinaire d'études religieuses

Théologiques

\section{Pour des initiatives continentales ou régionales}

\section{François-Xavier Amherdt}

Volume 27, numéro 2, 2019

Une décentralisation salutaire de l’Église catholique?

URI : https://id.erudit.org/iderudit/1069135ar

DOI : https://doi.org/10.7202/1069135ar

Aller au sommaire du numéro

Éditeur(s)

Institut d'études religieuses de l’Université de Montréal

ISSN

1188-7109 (imprimé)

1492-1413 (numérique)

Découvrir la revue

Citer cet article

Amherdt, F.-X. (2019). Pour des initiatives continentales ou régionales. Théologiques, 27(2), 123-136. https://doi.org/10.7202/1069135ar

\section{Résumé de l'article}

Si le pape François ne désire pas « remplacer les épiscopats locaux dans le discernement de toutes les problématiques qui se présentent sur leurs territoires » (Evangelii gaudium, n. 16), c'est qu'il souhaite renouer avec la pratique en cours à l'époque antique et patristique des initiatives continentales ou régionales. Une telle « décentralisation salutaire » réclamée par le souverain pontife argentin peut être réfléchie et encouragée par les Facultés de théologie à travers le monde, conçues par le même François comme des " laboratoires culturels providentiels " (Veritatis gaudium, n. 3). Ecclésiologiquement, elle s'appuie sur la nécessaire inculturation diversifiée de l'Évangile déjà envisagée par ses prédécesseurs Paul VI, Jean-Paul II et Benoît XVI, notamment à propos des formes de piété populaire spécifiques aux régions du globe. Elle peut s'exercer de manière instituée par la tenue encore plus régulière de Synodes régionaux ou continentaux, dont les options en matière de théologies des sacrements, des ministères ou des structures pourraient jouir d'une certaine autonomie - comme c'est d'ailleurs déjà le cas entre les Églises catholiques de rite oriental et latin.
Ce document est protégé par la loi sur le droit d'auteur. L'utilisation des services d’Érudit (y compris la reproduction) est assujettie à sa politique d'utilisation que vous pouvez consulter en ligne.

https://apropos.erudit.org/fr/usagers/politique-dutilisation/ 


\title{
Pour des initiatives continentales ou régionales
}

\author{
François-Xavier AMHERDT" \\ Théologie pastorale \\ Université de Fribourg (Suisse)
}

\begin{abstract}
J'ai accepté avec plaisir l'invitation des Pères synodaux à rédiger la présente Exhortation. En le faisant, je recueille la richesse des travaux du Synode. J'ai aussi consulté différentes personnes, et je compte en outre exprimer les préoccupations qui m'habitent en ce moment concret de l'œuvre évangélisatrice de l'Église. Les thèmes liés à l'évangélisation dans le monde actuel qui pourraient être développés ici sont innombrables. Mais j'ai renoncé à traiter de façon détaillée ces multiples questions qui doivent être l'objet d'étude et d'approfondissement attentif. Je ne crois pas non plus qu'on doive attendre du magistère papal une parole définitive ou complète sur toutes les questions qui concernent l'Église et le monde. Il n'est pas opportun que le Pape remplace les Épiscopats locaux dans le discernement de toutes les problématiques qui se présentent sur leurs territoires. En ce sens, je sens la nécessité de progresser dans une «décentralisation» salutaire. (François 2013, n. 16)
\end{abstract}

\footnotetext{
François-Xavier Amherdt est professeur de théologie pastorale, pédagogie religieuse et homilétique à l'Université de Fribourg (Suisse), ainsi que prêtre du diocèse de Sion (Valais, Suisse). Ancien vice-directeur du séminaire et vicaire épiscopal de son diocèse, il a été dix ans curé-doyen de Sierre et Noës, puis directeur de l'Institut romand de Formation aux Ministères à Fribourg. Il est co-responsable du Comité italo-helvétique de la rédaction et directeur-adjoint de Lumen Vitae. Ses principaux domaines de recherches sont: l'herméneutique biblique de Paul Ricœur en exégèse catholique, rhétorique et homilétique, l'animation biblique de la pastorale, la spiritualité des agents pastoraux, le développement de formes de catéchèse mystagogique. Il a récemment publié: (2019) F.-X. Amherdt, R. Lacroix, dir. (Équipe européenne de catéchèse), La conversion. L'acte, le processus, l'accompagnement, Saint-Maurice, Saint-Augustin (Perspectives pastorales 12); (2019) F.-X. AmHerdt et al., dir., S'ouvrir à l'Autre qui appelle, Saint-Maurice, Saint-Augustin (Les Cahiers de l'ABC 7).
} 


\section{Des réserves pontificales}

Selon cette déclaration tirée de l'exhortation La joie de l'Évangile (Evangelii gaudium, citée EG), l'exercice pontifical de la catholicité que prône le pape François est donc en quelque sorte limité en «qualité » et en «extensibilité ». Il ne prétend pas traiter l'ensemble des thématiques envisageables et entend en confier certaines à des théologiens et penseurs qui peuvent poursuivre la réflexion. Il ne souhaite pas non plus se substituer aux Conférences épiscopales locales et leur octroie la possibilité de discerner ce qu'il conviendrait de retenir et d'opérer en chacun de leurs contextes.

Bienheureux retrait du souverain pontife! Heureuse réserve de l'évêque de Rome! Sans doute s'inscrivent-ils dans la ligne de l'invitation que le pape Jean-Paul II avait déjà lancée dans le document sur l'œuménisme, $U t$ unum sint (Jean-Paul II 1995), y compris aux frères et sœurs des autres Églises chrétiennes, à l'aider à mieux exercer son ministère de communion universelle ${ }^{1}$.

\section{Perspectives de l'article}

La présente contribution tente d'explorer de manière prospective sur quelles bases théologiques et dans quelles directions une telle «décentralisation salutaire » pourrait peut-être advenir. Il ne s'agit en rien d'une mise en cause du Magistère romain. Bien au contraire, l'article cherche à commenter les propos mêmes du souverain pontife argentin cités en exergue et à montrer quelques-unes des voies qui pourraient s'offrir pour une mise en œuvre effective de ce vœu pontifical.

Nous commencerons par examiner en quoi les Facultés de théologie pourraient servir de "laboratoires culturels providentiels », en vue d'étudier les modalités d'une pareille décentralisation. Puis nous dégagerons quelques fondements ecclésiologiques à travers la nécessaire inculturation diversifiée de la Bonne Nouvelle, déjà souhaitée par les prédécesseurs de François sur le siège de Pierre. Nous le ferons notamment à travers la prise en compte réfléchie et cadrée des formes de piété populaire spécifiques aux régions et continents. Nous en arriverons donc à plaider en faveur d'une relative «autonomie» des Conférences épiscopales et pour la tenue régulière de Synodes continentaux, en tant que moyens de réalisation concrète de la décentralisation appelée de ses vœux par l'actuel évêque de Rome.

1. Voir, à propos de la recherche commune en vue de la communion catholique, Amherdt et al. (2013, n. 38). 
Nous nous risquerons alors, d'une façon que certains jugeront audacieuse, à inventorier quelques-unes des problématiques en cours, au sein desquelles une différenciation disciplinaire pourrait être prise en considération, en commençant par les plus envisageables selon les orientations actuelles. Il s'agirait de commencer par explorer les ouvertures déjà existantes et de ne pas perdre de vue les nécessités imposées par le dialogue œcuménique. Nous ne préjugeons pas de l'aboutissement effectif de nos suggestions et nous nous confions au discernement du sensus fidei du peuple de Dieu et de ses responsables².

\section{Des laboratoires culturels providentiels}

La visée de «décentralisation salutaire » esquissée par EG se retrouve également au cœur de la déclaration sur les études ecclésiastiques Veritatis gaudium (VG) (François 2017). Dans le préambule de celle-ci, le pontife argentin encourage les Facultés et Instituts de théologie partout dans le monde à œuvrer et continuer d'œuvrer, pour chacun d'eux, en tant que «laboratoire culturel providentiel» (n. 3). Cela signifie qu'ils sont invités à déployer leurs recherches et leurs actions comme centres d'études scientifiques («laboratoires») pluri- et inter-disciplinaires ${ }^{3}$, insérés dans le dynamisme spirituel de l'histoire du salut («providentiels») et profondément ancrés dans le contexte spatiotemporel où ils évoluent ( «culturels»), un appel valable pour les professeurs comme pour les étudiants, dont la pluralité est souvent réjouissante ${ }^{4}$.

Le résultat peut en être le déploiement encore plus marqué d'une pluralité de formes théologiques, à l'intérieur de la communion de foi catholique, mises en réseau les unes aux autres, en dialogue avec les Églises locales et le Magistère. Et nous pouvons espérer que les orientations disciplinaires qui devraient effectivement en découler soient à l'avenir plus souples et décentralisées que les normes préconisées par le récent docu-

2. Voir, à ce propos, l'important document de la Commission théologique internationale (2014); aussi: Bonino (2014a et 2014b); Butler (2014); McPartlan (2014).

3. Selon les quatre principes guidant l'évolution des études théologiques $(V G, n .4)$ : a) pour une théologie spirituelle et kérygmatique; b) favorisant le dialogue aux différents niveaux; c) encourageant la pluri- et l'interdisciplinarité; d) ainsi que le travail en réseau avec les autres Facultés.

4. À la Faculté de théologie bilingue de l'Université de Fribourg, où nous avons la joie d'enseigner la théologie pastorale, la pédagogie religieuse et l'homilétique, les enseignants proviennent d'une dizaine de pays et on compte plus de 50 nationalités différentes chez les étudiants et doctorants. C’est une «Église catholique» en miniature. 
ment, finalement assez nettement «romano-centrées » encore et en quelque sorte déconnectées des prolégomènes ( Préambule», n. 1 à 6). En effet, on a comme l'impression que ces premiers numéros sont issus de la plume même du pape actuel, puisque s'y retrouvent plusieurs de ses thématiques favorites, telles la théologie au service de la vie ecclésiale et de la justice sociale dans le monde de ce temps, dans la ligne de Gaudium et spes (GS). Au contraire, les «Normes» (articles 1 à 94) sentent plutôt «la patte» des Congrégations vaticanes ad hoc ${ }^{5}$ et reproduisent assez fidèlement les prescriptions déjà valables antérieurement, en ajoutant même des exigences supplémentaires, comme l'obtention pour les doyen (ne) s des Facultés du nibil obstat (voir article 18), alors qu'ils (elles) en bénéficient déjà au moment de la reconnaissance de leur nomination en tant que professeurs. De plus, il n'est guère tenu compte des conditions particulières que les Facultés canoniques intégrées dans des Universités d'État doivent honorer et qui peuvent varier d'un pays à l'autre.

Le contraste et la relative non-cohérence entre les deux volets de $V G$ sont en tout cas «révélateurs» (au sens photographique du terme, soit de révéler les couleurs des négatifs) des développements à accomplir, déjà au cœur de la curie romaine, en vue de la «salutaire décentralisation » voulue par le souverain pontife!

\section{Une salutaire inculturation}

Si décentralisation il convient d'y avoir, en fonction des diverses régions dont se compose l'Église catholique, c'est bien sûr au nom même de l'ecclésiologie de communion de Vatican $\mathrm{II}^{6}$, selon laquelle en chaque diocèse se réalise (subsistit in, n. 8) la plénitude de l'Église. Les évêques, qui sont convoqués après leur nomination et ordination dans la Ville éternelle pour y recevoir un cours d'initiation à la mise en œuvre harmonieuse de leur fonction épiscopale, ne sont pas des "préfets départementaux » ${ }^{7}$. La marge

5. Voir la déclaration finale du Fakultätentag des Facultés de théologie germanophones des 31 janvier - 2 février 2019 à Siegburg: «Erklärung der Vollversammlung des Katholisch-Theologischen Fakultätentags ", qui partage le même avis que nous (<kthf.de/wp-content/uploads/2015/10/2019-02-02-Erk1 \% C3\% A4rung-KThF-zuVeritatis-gaudium.pdf $>$ ).

6. Principalement exprimée dans la Constitution dogmatique sur l'Église Lumen gentium (1964).

7. Ou «cantonaux », dirions-nous dans la Confédération helvétique, dont le modèle de fonctionnement fédéraliste effectif, laissant une très grande autonomie à chacun des vingt-six cantons par rapport au pouvoir central de Berne et aux Offices nationaux, 
de manœuvre qui leur revient théologiquement pourrait davantage se traduire dans les faits et les textes législatifs.

Car il y va de la crédibilité de l'inculturation, dont les saints Paul VI ${ }^{8}$ et Jean-Paul ${ }^{9}$ II avaient déjà fait l'un de leurs leitmotivs pour l'acte d'annonce de la Bonne Nouvelle, afin que cela ne reste pas un vœu pieux. Le pontife actuel y consacre de très belles pages qui peuvent jouer le rôle de points de repères pour les nécessaires contextualisations de l'évangélisation (n. 115-118, «Un peuple aux multiples visages » $)^{10}$ :

Quand une communauté accueille l'annonce du salut, l'Esprit Saint féconde sa culture avec la force transformante de l'Évangile. De sorte que, comme nous pouvons le voir dans l'histoire de l'Église, le christianisme n'a pas un modèle culturel unique, mais "tout en restant pleinement lui-même, dans l'absolue fidélité à l'annonce évangélique et à la tradition ecclésiale, il revêtira aussi le visage des innombrables cultures et des innombrables peuples où il est accueilli et enraciné ${ }^{11}$. Chez les divers peuples, qui expérimentent le don de Dieu selon leur propre culture, l'Église exprime sa catholicité authentique et montre "la beauté de ce visage multiforme ${ }^{12}$. Dans les expressions chrétiennes d'un peuple évangélisé, l'Esprit Saint embellit l'Église, en lui indiquant de nouveaux aspects de la Révélation et en lui donnant un nouveau visage. Par l'inculturation, l'Église «introduit les peuples avec leurs cultures dans sa propre communauté ${ }^{13}$, parce que " toute culture offre des valeurs et des modèles positifs qui peuvent enrichir la manière dont l'Évangile est annoncé, compris et vécu » ${ }^{14}$. Ainsi, «l'Église, accueillant les valeurs des différentes cultures, devient la "sponsa ornata monilibus suis", "l'épouse qui se pare de ses bijoux" (cf. Is 61,10).»"15

pourrait peut-être partiellement inspirer les modalités de gouvernement des instances vaticanes - sans compter le poids octroyé en Suisse aux autorités communales à la base, pour une véritable politique proche de la population, qui elle aussi serait susceptible de «donner à penser» pour la "pastorale de proximité » dans la joie de l'Évangile que revendique l'Exhortation apostolique EG.

8. Voir Evangelii nuntiandi (Paul VI 1975).

9. Voir Redemptoris missio (Jean-Paul II 1990).

10. Voir à ce sujet notre ouvrage Culture et foi en dialogue (Amherdt 2015).

11. EG, n. 116, cite ici la Lettre apostolique Novo millennio ineunte (Jean-Paul II 2001, n. 40).

12. Voir Novo millennio ineunte (Jean-Paul II 2001, n. 40).

13. EG, n. 116, cite la Lettre encyclique Redemptoris missio du même Jean-Paul II (1990, n. 52), et renvoie à son Exhortation apostolique Catechesi Tradendae, n. 53.

14. Citation de l'Exhortation apostolique post-synodale Ecclesia in Oceania (Jean-Paul II 2001, n. 16, citée EO).

15. Citation de l'Exhortation apostolique post-synodale Ecclesia in Africa (Jean-Paul II 1995, n. 61). 
Cette diversité culturelle bien comprise et intégrée ne menace pas l'unité de l'Église si elle est portée par l'Esprit Saint qui, œuvrant dans la communion du Père et du Fils, suscite une harmonie multiforme et une unité échappant à toute uniformité. "L'évangélisation reconnaît avec joie ces multiples richesses que l'Esprit engendre dans l'Église », poursuit François, premier pontife de l'histoire issu de l'hémisphère Sud. «Ce n'est pas faire justice à la logique de l'incarnation que de penser à un christianisme monoculturel et monocorde. » Puisque le message de l'Évangile est «transculturel, [...] il n'est pas indispensable d'imposer une forme culturelle particulière, aussi belle et antique qu'elle soit, avec la proposition de l'Évangile », lorsque de nouvelles cultures sont évangélisées (EG, n. 117).

Le pape sud-américain mentionne alors les déclarations des évêques du $5^{\text {e }}$ continent qui ont demandé que - chez eux comme partout ailleurs l'Église «fasse comprendre et présente la vérité du Christ en s'inspirant des traditions et des cultures de la région» et qui ont formulé le souhait que «tous les missionnaires travaillent en harmonie avec les chrétiens autochtones pour faire en sorte que la foi et la vie de l'Église soient exprimées selon des formes légitimes appropriées à chaque culture.» $(E O$, n. 17) De cette manière, conclut le pape jésuite, «[...] la foi ne peut pas être enfermée dans les limites de la compréhension et de l'expression d'une culture particulière ${ }^{16}$. Il est indiscutable qu'une seule culture n'épuise pas le mystère de la rédemption du Christ. » (EG, n. 118)

\section{Les formes de piété populaire}

Ces considérations sur la bienfaisante décentralisation inculturée de la prédication évangélique valent d'autant plus pour les formes de piété populaire, fort variables d'une région à l'autre, dont La joie de l'Évangile vante la force évangélisatrice (n. 122-126). Renvoyant notamment au document du CELAM, dit d'Aparecida (EG, n. 124) ${ }^{17}$, l'évêque de Rome souligne l'importance de cette "spiritualité (ou mystique) populaire », parlant d'une véritable «spiritualité incarnée dans la culture des simples ${ }^{18}$.

16. Il renvoie ici à l'autre Exhortation apostolique post-synodale Ecclesia in Asia (JeanPaul II, n. 20).

17. Auquel il a notablement contribué comme archevêque de Buenos Aires. Voir Ve Conférence générale de l'épiscopat latino-américain et des Caraïbes (2007, n. 262), et le discours devant la session inaugurale de cette conférence de Benoît XVI, n. 1, Acta Apostolicae Sedis 99, 2007, p. 446-447.

18. Document d'Aparecida, n. 263, cité par EG, n. 124. 
La religiosité populaire enrichit la foi reçue de nouvelles expressions éloquentes de "l'action missionnaire spontanée du Peuple de Dieu. Il s'agit d'une réalité en développement permanent où l'Esprit Saint est l'agent premier ${ }^{19}$. C'est ainsi que par les formes «symboliques» issues de la continuité affective présente dans la piété des baptisés, particulièrement des pauvres et des petits, le "peuple s'évangélise continuellement lui-même» $(E G, \text { n. } 122)^{20}$. Les expressions multiformes de la religiosité populaire à travers le monde doivent être considérées aussi comme de véritables «lieux théologiques» au service de la «force activement évangélisatrice» de la «nouvelle évangélisation» et méritent aussi d'être encouragées et fortifiées "pour approfondir le processus d'inculturation qui est une réalité jamais achevée » (EG, n. 126).

\section{Conférences épiscopales}

De ce point de vue, même si canoniquement elles ont un statut moins assuré et déterminé que chaque successeur des apôtres dans son diocèse, il devrait revenir aux Conférences épiscopales, nationales ou régionales, de déterminer quelles priorités ecclésiales, et donc disciplinaires, il s'agirait de mettre en œuvre dans leurs juridictions. On en a abondamment parlé à l'occasion du Synode des évêques d'Amazonie (octobre 2019) ${ }^{21}$ : lorsque le bien du peuple de Dieu le requiert, en l'occurrence l'accès à l'eucharistie, centre de la vie chrétienne, source et sommet de l'existence des fidèles, les conditions d'accès au ministère de présidence eucharistique pourraient être revues par les évêques locaux, d'entente avec l'évêque de Rome, de manière par exemple à ce que des hommes mariés, dont le témoignage éprouvé par les communautés elles-mêmes les recommande comme aptes à exercer un tel ministère de manière fidèle et fiable (des viri probati), soient ordonnés pour le sacerdoce, ainsi que cela se pratique déjà pour l'ordination de diacres permanents mariés. La discipline catholique orientale ne le prévoitelle pas du reste déjà ? Il ne s'agirait alors que d'une extension de ce droit particulier à d'autres régions que celles actuellement visées par cette pratique admise dans des Églises rattachées au Siège romain.

19. Renvoi à Jean-Paul II, Ecclesia in Asia, n. 21, dans EG, n. 122.

20. La joie de l'Évangile cite ici la III Conférence générale de l'épiscopat latino-américain et des Caraibes (1979, n. 450), ainsi que le Document d'Aparecida, n. 264.

21. Voir <www.vaticannews.va/fr/vatican/news/2019-10/synthese-document-finalsynode-amazonie.html>. 
Dans cette même perspective, on pourrait imaginer que les agents pastoraux, qu'ils soient diacres permanents ou laïcs, maintenant déjà associés selon le canon 517,2 à la charge pastorale de gouvernance d'une paroisse, en lien avec un "curé modérateur ", soient eux aussi ordonnés prêtres. Ou alors, en attendant que cela se concrétise juridiquement, on pourrait envisager que ces derniers puissent officiellement se voir attribuer le titre de "curés» (de jure), puisque c'est de facto la charge qu'ils exercent déjà en tant que Gemeindeleiter — " responsables de communautés » si les Conférences des évêques du lieu l'estiment opportun ${ }^{22}$.

\section{Synodes continentaux}

Cela impliquerait donc que soient réunis plus régulièrement des Synodes d'évêques par continent ou par région, et que soient établies sur le plan de l'Église universelle les compétences théologiques et canoniques qui leur reviendraient. Cela fonctionne déjà ainsi à bien des égards, par exemple à propos des modalités de concrétisation de l'unique ministère de diacre permanent - totalement pastoral, comme un "mini-vicaire» en certains endroits, complètement enraciné dans un milieu de vie et une activité professionnelle dans d'autres, ou encore totalement inexistant en plusieurs pays - ou celui d'agent pastoral laï — inconnu en quelques régions, et très répandu en d'autres, au point que leur nombre excède celui des ministres ordonnés en certains endroits.

Les sensibilités pastorales si diverses au sein de la catholicité - comme cela est apparu lors des Synodes sur la famille d'octobre 2014 et 2015 pourraient ainsi amener à des législations différenciées par continents (ou hémisphères), sur plusieurs sujets brûlants. Nous les énumérons dans l'ordre décroissant de probabilité d'évolution, compte tenu de la situation présente:

1. la place des femmes dans des postes à responsabilités ecclésiales ${ }^{23}$;

2. la prédication lors des célébrations eucharistiques faite à tour de rôle par les prêtres, les diacres et les laïcs, formés et mandatés pour cet important service communautaire ${ }^{24}$, puisque le canon 766 en

22. Je pense notamment à des diocèses de Suisse alémanique (avec leurs Gemeindeleiter laïcs), ou de la RDC, selon l'intuition du cardinal Malula (les mokambis).

23. Telle que souhaitée d'ailleurs par EG, n. 104.

24. Témoignent de la valeur de cet exercice les abondants paragraphes d'EG consacrés à l'homélie (n. 135-144) et à la préparation de la prédication (n. 145-159). 
prévoit déjà la possibilité occasionnelle, et que cela se réalise dans toutes les célébrations non sacramentelles (telles les funérailles ou les liturgies de la Parole en aumôneries) et lors de certaines célébrations sacramentelles en cas de délégation octroyée pour les célébrations (mariages et baptêmes) ${ }^{25}$;

3. la mise en œuvre de procédures tenant compte des législations civiles locales, pour le délicat traitement des si douloureux cas d'abus sexuels commis par des clercs;

4. la pratique d'une espèce de catéchuménat baptismal pour les parents demandant le sacrement pour leur nouveau-né, mais se sentant éloignés de la foi et de l'Église: présentation de l'enfant, formation catéchétique étalée dans le temps des parents;

5. l'octroi de la possibilité éthiquement admise pour de jeunes couples envisageant sérieusement le sacrement de mariage d'un temps de cohabitation prénuptial en vue d'approfondir et de mûrir leur décision;

6. l'accompagnement des couples vivant en des situations dites «irrégulières ${ }^{26}$, avec la possibilité dans certains cas, moyennant un discernement vécu en compagnie d'un pasteur, de laisser l'accès à la communion pour les divorcés remariés au choix des personnes concernées;

7. et donc la mise en place différenciée selon les régions d'une sorte de catéchuménat du mariage, comportant différentes sortes d'étapes: rituel à l'occasion de l'installation en commun du couple ou de l'union civile; fiançailles ecclésiales; première présentation du couple, s'il est distancé de l'Église; ou reconnaissance de la valeur de préparation évangélique du mariage traditionnel et coutumier;

8. la pastorale auprès des personnes homosexuelles, pouvant aller jusqu'à la reconnaissance d'un rituel d'accueil des couples de même sexe - sans qu'il s'agisse d'une bénédiction nuptiale ni d'une célébration sacramentelle;

9. l'octroi de la possibilité de déléguer le ministère de sacrements comme l'onction des malades ou la réconciliation — à des baptisés

25. Selon les canons 1112,$1 ; 861,2$. Voir à ce propos le chapitre 8 de notre ouvrage $L a$ joie de prêcher (Amherdt 2018, 234-237).

26. Voir à ce propos le chapitre 8 de Amoris laetitia (François 2016, n. 291-312, citée $A L)$. 
laïcs, hommes et femmes (ainsi qu'à des diacres permanents), dans le cadre de l'exercice de leur mandat d'accompagnement suivi des malades, des personnes en difficulté, en précarité, en grandes pauvretés; etc.

Dans l'ensemble de ces problématiques, la théologie en jeu demeurerait une, mais diversifiée, grâce au travail de la Commission théologique internationale approuvé par le Magistère romain. La possibilité serait alors laissée aux Conférences des évêques des diverses régions de l'actualiser d'une manière spécifique, selon les besoins locaux, suite à une réflexion mûrie aboutissant à des décisions prises lors de synodes (ou «conciles») continentaux, en présence du Saint-Père ou de son délégué. Ce serait en quelque sorte revenir à la pratique des conciles régionaux de l'Église antique et médiévale, dont les pouvoirs décisionnels étaient réels.

\section{Mieux exploiter les possibilités existantes}

La liste des quaestiones disputatae en Église, qui pourraient être partiellement réglées par des options canoniques décentralisées, serait bien sûr susceptible d'être étendue à l'infini. La mise en œuvre d'une telle pratique renforcerait l'exercice effectif de la synodalité ecclésiale à différents degrés $^{27}$, sans que les autres principes de la communion universelle et de la référence aux responsables hiérarchiques ne soient pour autant entamés ni bien sûr supprimés.

Des «galops d'essai » pourraient être entrepris pour des réalités où, selon les textes magistériels officiels, une telle possibilité existe déjà, mais n'est pas pleinement exploitée. Nous pensons par exemple aux ministères institués, actuellement réservés aux hommes et dans la plupart des pays aux candidats au diaconat et au presbytérat. Il est à noter que le décret Ministeria quaedam de Paul VI, révisant les voies d'accès aux ordinations, souhaitait déjà en 1972 les étendre à d'autres ministères institués que le lectorat (service de la table de la Parole) et que l'acolytat (service de la table de l'autel et des pauvres), et donc à des laïcs (femmes et hommes) non appelés au sacrement de l'ordre. Le Document final du Synode sur la Parole

27. Voir le document de la Commission théologique internationale (2018); aussi: <www. vatican.va/roman_curia/congregations/cfaith/cti_documents/rc_cti_20180302_ sinodalita_fr.html>. 
de Dieu de $2008^{28}$ avait d'ailleurs suggéré une extension du lectorat aux femmes, ce que Benoît XVI n'avait pas repris dans l'exhortation apostolique post-synodale Verbum Domini (Benoît XVI 2010).

Alors que certains diocèses ${ }^{29}$ ont exploité cette voie, créant même des écoles de formation en vue de l'obtention d'un ministère institué, la plupart des parties de l'Église ne l'ont pas du tout retenue. Un élargissement des institutions à des ministères de type diaconal — tels les aumôniers d'établissements hospitaliers ou scolaires, les chargés d'information et de formation, les responsables de services et mouvements - permettrait de retrouver la diversification ministérielle - qui existait dans les communautés des premiers siècles et qui s'est perdue progressivement dans la mesure où toutes les charges se sont concentrées sur la figure presbytérale -, et donnerait ainsi l'occasion d'associer davantage de femmes à des responsabilités liturgiquement et ecclésialement reconnues.

\section{Sur le plan œcuménique}

Une telle décentralisation salutaire à la fois valoriserait la variété des formes potentielles non concrétisées et débloquerait certaines problématiques actuellement dans l'impasse. Elle signifierait également des avancées en direction de nos frères et sœurs chrétiens qui tous pratiquent une autonomie locale plus importante que l'Église catholique - les Églises autocéphales orthodoxes, la myriade d’Églises réformées protestantes, anglicanes ou évangéliques. Inversement, cela donnerait le coup d'envoi à des réflexions chez nos partenaires œuméniques en direction d'un ministère de la communion, soit sur le plan local (de type épiscopal), soit au niveau universel (une primauté d'honneur attribuée au "patriarche de Rome» $)^{30}$.

Les voies synodales de la décentralisation - explorées par de nombreux synodes (ou démarches quasi synodales) diocésains ou régionaux, par quelques Synodes des évêques continentaux, et par l'institutionnalisation du Synode des évêques mondial régulier, en guise de forme d'aide au gouvernement du souverain pontife comme de chaque évêque et des Conférences épiscopales - s'avèrent prometteuses, malgré les réflexes de repli

28. Voir : <fr.zenit.org/articles/texte-integral-du-message-du-synode-sur-la-parole-dedieu>.

29. Comme Bâle avec le Jura pastoral en Suisse, Palerme en Sicile et d'autres en Italie.

30. Voir quelques-unes de nos suggestions à l'adresse autant de notre Église que des autres communautés ecclésiales, dans Amherdt et al. (2013). 
centripète constaté chez des représentants de la curie et même chez de (jeunes) agents pastoraux.

La métaphore paulinienne du corps (voir 1 Co 12), très souvent lue dans le sens de l'unité conférée par le même Esprit, le même souffle agissant dans l'ensemble des membres, vaut tout autant pour mettre en exergue les différences existant entre les bras, les genoux, l'intestin, les oreilles ou les orteils. C'est à condition que le sang irrigue les extrémités ou périphéries, dirait François (voir EG, n. 20), que le corps se porte au mieux. Si loin du centre, mais si proche du cœur, c'est-à-dire du Christ et de la Trinité sainte: ainsi sont appelées les Églises locales à faire preuve de créativité et d'innovation pour le bien de l'ensemble du corps ecclésial. C'est le pape lui-même qui le demande et l'attend!

\section{Références}

Ve CONFÉRENCE GÉNÉRALE DE L'ÉPISCOPAT LATINO-AMÉRICAIN ET DES Caraïbes (2007), Document d'Aparecida (29 juin 2007); disponible sur: <www.celam.org/aparecida/Frances.pdf >.

Amherdt, F.-X (2015), Culture et foi en dialogue, coll. «Vatican II pour tous ", Paris, Médiaspaul.

(2018), La joie de prêcher. Petit manuel, Saint-Maurice, SaintAugustin (Perspectives pastorales 10).

Amherdt, F.-X., M. Hoegger, P. GonZalez et H. Paik (2013), dir., Vers une catholicité oecuménique?, Fribourg, Academic Press (Théologie pratique en dialogue 38).

Benoît XVI (2010), Verbum Domini. Exhortation apostolique postsynodale sur la Parole de Dieu dans la vie et dans la mission de l'Église, Rome.

Bonino, S.-T. (20I4a), "Pour lire le document Le "sensus fidei" dans la vie de l'Église», <www.vatican.va/roman_curia/congregations/cfaith/ cti_documents/rc_cti_20140610_cerf-sensus-fidei_fr.html>.

(2014b), «Il fiuto delle pecore. Un nouveau document de la Commission théologique internationale: "Le sensus fidei dans la vie de l'Église" ", <www.vatican.va/roman_curia/congregations/cfaith/ cti_documents/rc_cti_20140610_bonino-sensus-fidei_fr.html>.

Butler, S. (2014), "Sensus fidei, Chapters One and Two », disponible sur: <www.vatican.va/roman_curia/congregations/cfaith/cti_documents/ rc_cti_20140610_butler-sensus-fidei_en.html>. 
COMMISSION THÉOlogiQue INTERNATIONALE (2014), "Le sensus fidei dans la vie de l'Église", Rome; disponible sur: <www.vatican.va/roman curia/congregations/cfaith/cti_documents/rc_cti_20140610_sensusfidei_fr.html>.

(2018), La synodalité dans la vie et dans la mission de l'Église, Rome, disponible sur: <www.vatican.va/roman_curia/congregations/ cfaith/cti_documents/rc_cti_20180302_sinodalita_fr.html>.

FrançoIs (2013), Evangelii gaudium. Exhortation apostolique sur l'annonce de l'Évangile dans le monde d'aujourd'hui, Rome.

(2016), Amoris laetitia. Exhortation sur l'amour dans la famille, Rome.

(2017), Veritatis gaudium, Constitution apostolique sur les Universités et les Facultés ecclésiastiques, Rome.

Jean-Paul II (1979), Exhortation apostolique Catechesi Tradendae, Rome.

(1990), Redemptoris missio. Lettre encyclique sur la valeur permanente du précepte missionnaire, Rome.

(1995a), Ut unum sint. Lettre encyclique sur l'engagement øecménique, Rome. Rome.

(1995b), Exhortation apostolique post-synodale Ecclesia in Africa,

(1999), Exhortation apostolique post-synodale Ecclesia in Asia, Rome.

(2001a), Lettre apostolique Novo millennio ineunte, Rome.

(2001b), Exhortation apostolique post-synodale Ecclesia in Oceania, Rome.

McPartlan, P. (2014), "The Sensus Fidei. A vital Resource for the Church», disponible sur: <www.vatican.va/roman_curia/congregations/cfaith/ cti_documents/rc_cti_20140625_mcpartlan-sensus-fidei_en.html>.

Paul VI (1975), Evangelii nuntiandi, Exhortation apostolique sur l'évangélisation dans le monde moderne, Rome.

VATICAN II (1964), Constitution dogmatique sur l'Église Lumen gentium, Rome.

(1965), Gaudium et spes. Constitution pastorale sur l'Église dans le monde de ce temps, Rome. 


\section{Résumé}

Si le pape François ne désire pas «remplacer les épiscopats locaux dans le discernement de toutes les problématiques qui se présentent sur leurs territoires" (Evangelii gaudium, n. 16), c'est qu'il souhaite renouer avec la pratique en cours à l'époque antique et patristique des initiatives continentales ou régionales. Une telle «décentralisation salutaire » réclamée par le souverain pontife argentin peut être réfléchie et encouragée par les Facultés de théologie à travers le monde, conçues par le même François comme des "laboratoires culturels providentiels" (Veritatis gaudium, n. 3). Ecclésiologiquement, elle s'appuie sur la nécessaire inculturation diversifiée de l'Évangile déjà envisagée par ses prédécesseurs Paul VI, Jean-Paul II et Benoît XVI, notamment à propos des formes de piété populaire spécifiques aux régions du globe. Elle peut s'exercer de manière instituée par la tenue encore plus régulière de Synodes régionaux ou continentaux, dont les options en matière de théologies des sacrements, des ministères ou des structures pourraient jouir d'une certaine autonomie — comme c'est d'ailleurs déjà le cas entre les Églises catholiques de rite oriental et latin.

\section{Abstract}

This article suggests that Pope Francis wishes to reconnect with the practice of continental and regional initiatives that were current in Antiquity and patristic times. Such a "salutary decentralization" claimed by the Argentine Pontiff can be thought and encouraged by Faculties of theology throughout the world. Ecclesiologically, it is grounded on a diversified inculturation of the Gospel that his predecessors, Paul VI, John Paul II, and Benedict XVI, already considered, especially some forms of popular piety that are specific to the regions of the world. It can be implemented by holding more regularly regional or continental synods that could be more autonomous in terms of the theology of sacraments, the ministries, and the structures - as it is already the case between the Eastern and Latin Churches. 